\title{
Commentary: All aboard the transcatheter express-Road map for surgeons to develop expertise in catheter- based treatment of structural heart disease
}

\author{
Grayson H. Wheatley III, MD
}

\author{
From Siragusa Vascular, LLC, Nashville, Tenn. \\ Disclosures: Author serves as a consultant for Medtronic, Terumo Aortic, and Ethicon. \\ Received for publication Jan 25, 2019; accepted for publication Jan 28, 2019; available ahead of print Feb 26, \\ 2019. \\ Address for reprints: Grayson H. Wheatley III, MD, Siragusa Vascular, LLC, 28 White Bridge Pike, Suite 200, \\ Nashville, TN 37205 (E-mail: ghw3rd@gmail.com). \\ J Thorac Cardiovasc Surg 2019;157:2377-8 \\ $0022-5223 / \$ 36.00$ \\ Copyright $(\underset{2}{ } 2019$ by The American Association for Thoracic Surgery \\ https://doi.org/10.1016/j.jtcvs.2019.01.103
}

It is not the mountain we conquer, but ourselves. —-Sir Edmund Hillary (1919-2008)

On Friday May 29, 1953, Sir Edmund Hillary become the first person to scale Mount Everest, as a testament to the value of shear will power and extreme endurance in conquering new challenges. He credits much of the success of that expedition to the expert assistance and detailed knowledge of the Mount Everest terrain of his faithful Sherpa mountain guide, Tenzing Norgay. The positive impact of having a knowledgeable guide who has detailed knowledge of the environment cannot be overestimated when seeking to conquer a challenging endeavor.

For surgeons, the acquisition of high-level catheter-based training, along with the ability to function as a meaningful contributor to the heart team, can be a daunting process. Increasingly, the value of including surgeons on the heart team is being questioned, not only for transcatheter aortic valve repair procedures but also for other advanced transcatheter interventions, such as transcatheter mitral valve replacement. In this issue of the Journal, Nguyen and colleagues ${ }^{1}$ share their road map for acquiring the technical skills necessary for performing catheter-based interventions for structural heart disease (SHD) and also detail the cognitive expertise that is required of surgeons to maintain their involvement on the heart team. We would all benefit from heeding their advice, because all of them are true visionaries who are willing to be our transcatheter Sherpa guides. They have mapped out the best ascent route up the transcatheter skills mountain and can expertly guide us to the summit.

Because of a current lack of a defined catheter skills training curriculum, current and future generations of cardiac surgeons are in danger of not possessing the necessary skill set to contribute to the heart team. Having a road map and a detailed breakdown of the components involved with transcatheter heart valve therapies thus helps ensure that

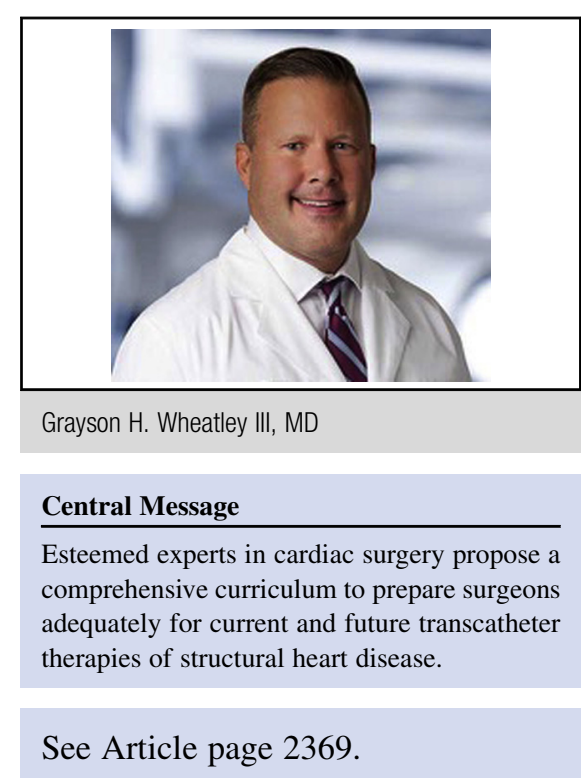

surgeons continue to play a valuable role on the heart team for the benefit of patients.

The transcatheter curriculum provides an invaluable resource for cardiac surgeons of all levels. Cardiac surgeons in training who are interested in pursuing a career specializing in the full spectrum of therapies available for the treatment of SHD can benefit by having a clearer understanding of the additional training required to acquire catheter-based skills. In addition, Nguyen and colleagues ${ }^{1}$ intend for this road map to be a resource for residency program directors to assist them in incorporating transcatheter training into their programs' curricula. Expertise in transcatheter valve procedures requires additional fellowship-level training, but ideally having expanded exposure to catheter-based training opportunities during residency would better prepare cardiac surgeons for a range of catheter-based procedures, including nonstructural heart-related procedures such as thoracic endovascular aortic repair. ${ }^{2}$

Early career cardiac surgeons can also benefit from this road map as they seek to establish themselves professionally in their practices. Whether treating SHD is a significant part of their clinical practice or is only a fraction of their clinical focus, possessing catheter-based training gives these young clinicians the best chance to being able to provide a knowledgeable surgical voice to the heart team. 
Catheter-based skills, such as transseptal puncture, are not commonly possessed by cardiac surgeons or even part of their training, yet they are critical skill sets for many of the current and future transcatheter solutions for treating SHD. Certainly, cardiac surgeons bring an important perspective to the heart team because of their unique understanding of the intricacies of SHD and have a sophisticated knowledge about appropriate patient selection.

Future transcatheter technologies will continue to emerge to address a host of new structural heart problems, and each of these technologies will require increasingly advanced catheter-based skills. As such, without focusing on new transcatheter training curricula, cardiac surgeons will increasingly become less able to master these technologies and risk becoming marginalized on the heart team. Nguyen and colleagues ${ }^{1}$ have provided an intelligent and thoughtful call to action for cardiac surgeons and cardiac surgery training programs. It is time for cardiac surgeons to listen closely and follow this road map so that they are able to punch their tickets on the transcatheter skills express.

\section{References}

1. Nguyen TC, Tang GH, Nguyen S, Forcillo J, George I, Kaneko T, et al. The train has left: can surgeons still get a ticket to treat structural heart disease? J Thorac Cardiovasc Surg. 2019;157:2369-76.e2.

2. Vardas PN, Stefanescu Schmidt AC, Lou X, Goldstone AB, Pattakos G, Fiedler AG, et al. Current status of endovascular training for cardiothoracic surgery residents in the United States. Ann Thorac Surg. 2017;104:1748-54. 